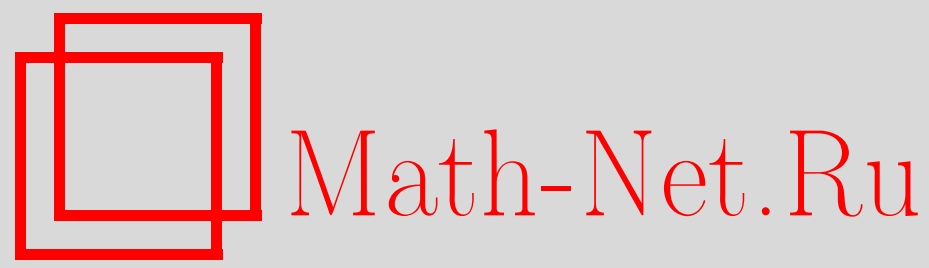

И. В. Протасов, Асимметрично разложимые абелевы группы, Матем. заметки, 1996, том 59, выпуск $3,468-471$

DOI: https://doi.org/10.4213/mzm1740

Использование Общероссийского математического портала MathNet.Ru подразумевает, что вы прочитали и согласны с пользовательским соглашением

http: //www . mathnet.ru/rus/agreement

Параметры загрузки:

IP : 54.198 .64 .247

26 апреля 2023 г., $17: 28: 48$

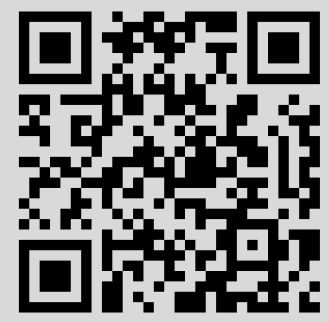




\section{АСИММЕТРИЧНО РАЗЛОЖИМЫЕ АБЕЛЕВЫ ГРУППЫ}

\section{И.В. Протасов}

Бесконечную абелеву группу $G$ назовем асимметрично разложимой, если сушествует такое разбиение группы $G$ на два подмножества $A_{1}, A_{2}$, что $(g+S) \cap A_{1} \neq \varnothing,(g+S) \cap A_{2} \neq \varnothing$ для любого элемента $g \in G$ и бесконечного симметричного подмножества $S \subseteq G$. Подмножество $S$ симметрично, если $S=-S$. Цель заметки - доказательство следующей теоремы.

ТЕОрема. Группа $G$ асимметрично разложсима тогда и только тогда, когда $G$ принадлехсит одному из двух классов групп:

(1) счетные периодические группы с конечным числом әлементов порядка 2

(2) $\mathbb{Z} \times K$, әде $K-$ конечная әруппа.

Сначала введем некоторые определения и обозначения. Фиксируем разбиение $G=A_{1} \cup A_{2}$ произвольной бесконечной группы $G$. Два элемента группы назовем одноцветными, если они принадлежат одному подмножеству разбиения. Подмножество группы одноцветно, если оно содержится в одном из подмножеств разбиения. Элементы $g_{1}, g_{1} \in G$ симметричны относительно элемента $a \in G$, если $g_{1}+g_{2}=2 a$. Для элемента $a \in G$ и подмножества $A \subseteq G$ положим

$$
\begin{aligned}
& S(a)=\{g \in G, 2 a-g \text {-одноцветные элементы }\}, \\
& S(A)=\cup\{S(a): a \in A\} .
\end{aligned}
$$

Доказательство теоремы составим из следующих утверждений.

1. Если $g \notin S(0)$ и $g \notin(-S(a))$, то элементы $g, g+2 a$ одночветны.

Пусть для определенности $g \in A_{1}$. Так как $g \notin S(0)$, то $(-g) \in A_{2}$. Поскольку $(-g) \notin S(a)$, то $2 a+g \in A_{1}$.

2. Пусть $H$ - подгруппа әруппь $G$, порохсденная некоторым подмножсеством $A \subseteq G, 2 H=\{2 h: h \in H\}$. Ecли $(g+2 H) \cap(S(0) \cup(-S(a)))=\varnothing$, то смежный класс $g+2 H$ одноцветный.

Возьмем произвольный элемент $b \in g+2 H$ и индукцией по числу $m$ покажем, что элементы $b$ и $b+2 a_{1}+\cdots+2 a_{m}$ одноцветны для любых $a_{1}, \ldots, a_{m} \in A$. Для $m=1$ это следует из утверждения 1 . Рассмотрим элемент $b+2 a_{1}+\cdots+2 a_{m}+2 a_{m+1}$. По предположению индукции элементы $b, b+2 a_{1}+\cdots+2 a_{m}$ одноцветны. Так как $b+2 a_{1}+\cdots+2 a_{m} \in g+2 H$ и $S\left(a_{m+1}\right) \subseteq S(A)$, то по условию $b+2 a_{1}+\cdots+2 a_{m} \notin$ $\left(S(0) \cup\left(-S\left(a_{m+1}\right)\right)\right)$. Применяя утверждение 1 , заключаем, что элементы $b+2 a_{1}+\cdots+2 a_{m}, b+2 a_{1}+\cdots+2 a_{m+1}$ одноцветны.

Произвольный элемент $c \in g+2 H$ представим в виде $c=g+2 a_{1}+\cdots+2 a_{m}$ $-2 a_{1}^{\prime}-\cdots-2 a_{k}^{\prime}$, где $a_{1}, \ldots, a_{m}, a_{1}^{\prime}, \ldots, a_{k}^{\prime} \in A$. По доказанному выше пары элементов $g, g+2 a_{1}+\cdots+2 a_{m}$ и $c, c+2 a_{1}^{\prime}+\cdots+2 a_{k}^{\prime}$ одноцветны. Так как $g+2 a_{1}+\cdots+2 a_{m}=c+2 a_{1}^{\prime}+\cdots+2 a_{k}^{\prime}$, то элементы $g$ и $c$ одноцветны. Таким образом, все элементы смежного класса $g+2 H$ имеют тот же цвет, что и элемент $g$. 
3. Если асимметрично разлохимая әруппа $G$ содерхит бесконечную конечно порохсденую подгруппу $H$, то факторгруппа $G / H$ конечна.

Пусть $G=A_{1} \cup A_{2}$ - асимметричное разбиение группы $G$. Очевидно, что подмножество $S(a)$ конечно для любого элемента $a \in G$. Поэтому конечно и подмножество $S(0) \cup(-S(A))$, где $A$ - конечное множество образующих подгруппы $H$. Возьмем произвольный элемент $g \in G$. Так как подгруппа $2 H$ бесконечна, то смежный класс $g+2 H$ не может быть одноцветньм. Из утверждения 2 следует, что $g \in((S(0) \cup(-S(A)))+H)$.

4. Асимметрично разлохимая группа содержит лишь конечное число элементов порядка 2.

Действительно, всякое подмножество группы, состоящее из элементов порядка 2, симметрично.

\section{5. Асимметрично разлохимая әруппа счетна.}

Допустим противное и фиксируем асимметричное разбиение $A_{1} \cup A_{2}$ несчетной группы $G$. Выделим счетную подгруппу $H$ группы $G$. Так как подмножество $S(a)$ конечно для любого элемента $a \in G$, то подмножество $S(0) \cup(-S(H))$ счетно. Поскольку группа $G$ несчетна, то найдется такой элемент $g \in G$, что $(S(0) \cup$ $(-S(H))) \cap(g+2 H)=\varnothing$. По утверждению 2 смежный класс $g+2 H$ одноцветен, а по утверждению 4 подгруппа $2 H$ бесконечна. Получили противоречие с тем, что $A_{1} \cup A_{2}$ - асимметричное разбиение группы $G$.

6. Счетная периодическая әруппа $G$ с конечным числом элементов порядка 2 асимметрично разложсима.

Заметим, что факторгруппа $G / H$ по любой конечной подгруппе $H$ имеет лишш конечное число элементов порядка 2 . Поэтому группу $G$ можно представить в виде объединения такой возрастающей цепочки конечных подгрупп

$$
G_{0} \subset G_{1} \subset G_{2} \subset \cdots \subset G_{n} \subset G_{n+1} \subset G_{n+2} \subset \cdots
$$

что $G_{n+1} / G_{n}$ содержит все элементы порядка 2 группы $G_{n+2} / G_{n}$. Поэтому для подходящего подмножества $K_{n} \subset\left(G_{n+2} \backslash G_{n+1}\right)$ существует следующее разложение подмножества $G_{n+2} \backslash G_{n+1}$ на смежные классы по подгруппе $G_{n}$ :

$$
G_{n+2} \backslash G_{n+1}=\left(K_{n}+G_{n}\right) \cup\left(-K_{n}+G_{n}\right) .
$$

Положим $A_{1}=\cup\left\{K_{n}+G_{n}: n<\omega\right\}, A_{2}=G \backslash A_{1}$. Фиксируем произвольные элемент $g \in G$ и симметричное бесконечное подмножество $s \subseteq G$. Выберем такой номер $m$, что $g \in G_{m}$. Так как подмножество $S$ бесконечно, а подгруппа $G_{m}$ конечна, то найдется такой номер $n \geqslant m$, что $S \cap\left(G_{n+2} \backslash G_{n+1}\right) \neq \varnothing$. Пусть $a \in S \cap\left(G_{n+2} \backslash G_{n+1}\right)$ и для определенности $a \in K_{n}+G_{n}$. Тогда $a+g \in K_{n}+G_{n}$ и $g+a \in A_{1}$. С другой стороны, $(-a) \in\left(-K_{n}+G_{n}\right)$ и $(-a+g) \in\left(-K_{n}+G_{n}\right)$. Значит, $g-a \in A_{2}$. Таким образом, $(g+S) \cap A_{1} \neq \varnothing$ и $(g+S) \cap A_{2} \neq \varnothing$. 
7. Группа $G=\mathbb{Z} \times K$ асимметрично разложима для любой конечной zpynnbı $K$.

Элемент $(z, a)$ группы $G$ отнесем к подмножеству $A_{1}$, если $z \geqslant 0$, и положим $A_{2}=G \backslash A_{1}$. Фиксируем произвольные элемент $g=\left(z_{0}, a_{0}\right)$ группы $G$ и бесконечное симметричное подмножество $S \subseteq G$. Так как группа $K$ конечна, аподмножество $S$ бесконечно, то найдется такой элемент $(z, a) \in S$, что $z+z_{0}>0, z_{0}-z<0$. Тогда $g+(z, a) \in A_{1}, g-(z, a) \in A_{2}$. Значит, $(g+S) \cap A_{1} \neq \varnothing,(g+S) \cap A_{2} \neq \varnothing$.

Завершим доказательство теоремы. Если асимметрично разложимая группа $G$ содержит элемент бесконечного порядка, то $G$ принадлежит классу (2) по утверждению 3. Периодическая асимметрично разложимая группа принадлежит классу (1) по утверждениям 4, 5. Асимметричная разложимость групп из классов $(1),(2)$ вытекает из утверждений 6,7 .

ЗАМЕчАНИЕ 1. Для любого конечного разбиения бесконечной группы $G=A_{1} \cup \cdots \cup A_{m}$ и любого натурального числа $n$ найдутся такие элемент $g \in G$ и конечное симметричное подмножество $S \subseteq G$, что $|S|>n$ и $g+S \subseteq A_{i}$ для некоторого $i \leqslant m$. Действительно, если группа $G$ содержит бесконечную подгруппу конечной экспоненты, то по теореме Грэхема-Либа-Росчайльда [1] в одном из подмножеств разбиения содержится смежный класс по конечной подгруппе порядка $>n$. Если группа $G$ содержит элемент бесконечного порядка, то применима теорема Ван дер Вардена об арифметических прогрессиях. Наконец, если порядки конечных циклических подгрупп группы $G$ неограничены в совокупности, то также работает теорема Ван дер Вардена, но в следующей конечной форме. Для любых натуральных чисел $k, m$ найдется такое натуральное число $M$, что при любом раскрашивании отрезка $\{1, \ldots, M\}$ натуральных чисел в $m$ цветов найдется одноцветная арифметическая прогрессия длины $k$.

ЗАмЕчаниЕ 2. Для любого разбиения бесконечной группы $G$ на три подмножества $G=A_{1} \cup A_{2} \cup A_{3}$ найдется такое бесконечное симметричное подмножество $S$, что $S \cap A_{i}=\varnothing$ для некоторого $i \leqslant 3$. Положим $A_{k j}=\left\{g \in G: g \in A_{k}\right.$, $\left.-g \in A_{j}\right\}$. Поскольку $A_{k j}=-A_{j k}$, то подмножество $A_{k j} \cup A_{j k}$ симметрично. Так как группа $G$ бесконечна, то по крайней мере одно из этих подмножеств бесконечно. Выберем номер $i$, отличный от $j, k$. Тогда $\left(A_{k j} \cup A_{j k}\right) \cap A_{i}=\varnothing$.

ЗАмЕч АниЕ 3 . Предположим, что бесконечную группу $G$ можно разбить на конечное число подмножеств $G=A_{1} \cup \cdots \cup A_{m}$ так, что $g+S \not \subset A_{i}$ для любых $i \leqslant m$, элемента $g \in G$ и бесконечного симметричного подмножества $S \subseteq G$. Наименьшее число $m$, для которого такое разбиение существует, обозначим $\nu(G)$. Положим $\nu(G)=\infty$, если конечного разбиения группы $G$ с таким свойством не существует. Таким образом, $\nu(G)$ - это наименьшее число цветов, которыми можно раскрасить группу $G$ так, чтобы не было бесконечных одноцветных подмножеств, симметричных относительно некоторого элемента группы. Заметим, что $\nu(G)>1$ и $\nu(G)=2$ тогда и только тогда, когда группа $G$ асимметрично разложима. Автор располагает лишь следующей весьма скудной информацией о числах $\nu(G)$ :

$$
\nu\left(\mathbb{Z}^{2}\right)=3, \quad \nu\left(\mathbb{Z}^{n}\right) \leqslant n+1, \quad \nu\left(\mathbb{Q}^{n}\right) \leqslant 2(n+1), \quad \nu(\mathbb{R})>3 .
$$

Таким образом, автору неизвестны даже числа $\nu\left(\mathbb{Z}^{3}\right), \nu(\mathbb{Q}), \nu(\mathbb{R})$. 
ЗАмеч Ание 4 . В статье [2] бесконечная группа $G$ названа абсолютно разложимой, если ее можно разбить на два подмножества, плотные в любой недискретной групповой топологии на $G$. Очевидно, что любая асимметрично разложимая группа абсолютно разложима. Однако, класс абсолютно разложимых групп существенно шире класса асимметрично разложимьх групп. Например, свободная группа любого ранга абсолютно разложима. Более того, не отвергнута гипотеза об абсолютной разложимости любой группы с конечным числом элементов порядка 2.

Киевский государственный университет

Поступило им. Тараса Шевченко 26.04 .95

\section{СПИСОК ЦИТИРОВАННОЙ ЛИТЕРАТУРЫ}

1. Грэхем Р. Начала теории Рамсея. М.: Мир, 1984. 2. Comfort W. W., Van Mill J. // Proc. Amer. Math. Soc. 1994. V. 120. № 3. P. 687-696. 